\title{
Automated registration of breast lesions in temporal pairs of mammograms for interval change analysis-local affine transformation for improved localization
}

\author{
Lubomir Hadjiiski, ${ }^{\text {a) }}$ Heang-Ping Chan, Berkman Sahiner, Nicholas Petrick, \\ and Mark A. Helvie \\ Department of Radiology, University of Michigan, Ann Arbor, Michigan 48109
}

(Received 15 August 2000; accepted for publication 9 April 2001)

\begin{abstract}
Analysis of interval change is important for mammographic interpretation. The aim of this study is to evaluate the use of an automated registration technique for computer-aided interval change analysis in mammography. Previously we developed a regional registration technique for identifying masses on temporal pairs of mammograms. In the current study, we improved lesion registration by including a local alignment step. Initially, the lesion position on the prior mammogram was estimated based on the breast geometry. An initial fan-shaped search region was then defined on the prior mammogram. In the second stage, the location of the fan-shaped region on the prior mammogram was refined by warping, based on an affine transformation and simplex optimization in a local region. In the third stage, a search for the best match between the lesion template from the current mammogram and a structure on the prior mammogram was carried out within the search region. This technique was evaluated on 124 temporal pairs of mammograms containing biopsyproven masses. Eighty-seven percent of the estimated lesion locations resulted in an area overlap of at least $50 \%$ with the true lesion locations and an average distance of $2.4 \pm 2.1 \mathrm{~mm}$ between their centroids. The average distance between the estimated and the true centroid of the lesions on the prior mammogram over all 124 temporal pairs was $4.2 \pm 5.7 \mathrm{~mm}$. The registration accuracy was improved in comparison with our previous study that used a data set of 74 temporal pairs of mammograms. This improvement in accuracy resulted from the improved geometry estimation and the local affine transformation. (C) 2001 American Association of Physicists in Medicine.
\end{abstract}

[DOI: 10.1118/1.1376134]

Key words: mammography, interval change, computer-aided diagnosis, breast cancer, affine transformation

\section{INTRODUCTION}

Mammography is currently the most effective method for early breast cancer detection. ${ }^{1,2}$ One of the important techniques used by radiologists in mammographic interpretation to detect developing malignancy is analysis of interval changes. ${ }^{3,4}$ A variety of computer-aided diagnosis (CAD) techniques have been developed to detect mammographic abnormalities and to distinguish between malignant and benign lesions. We are studying the use of CAD techniques to assist radiologists in interval change analysis.

Sallam et l. $^{5}$ have proposed a warping technique for mammogram registration based on manually identified control points. A mapping function was calculated for mapping each point on the current mammogram to a point on the prior mammogram. Brzakovic et al. ${ }^{6}$ have investigated a threestep method for comparison of the most recent and the prior mammograms. They first registered two mammograms using the method of principal axis, and partitioned the current mammogram using a hierarchical region-growing technique. Translation, rotation, and scaling were then used for registration of the partitioned regions. Vujovic et al. ${ }^{7}$ have proposed a multiple-control-point technique for mammogram registration. They first determined several control points independently on the current and prior mammograms based on the intersection points of prominent anatomical structures in the breast. A correspondence between these control points was established based on a search in a local neighborhood around the control point of interest.

The previous techniques depend on the identification of control points. However, because the breast is mainly composed of soft tissue that can change over time, there are no obvious landmarks on mammograms. The crossing line structures are often fibrous tissue from different depths of the breast which overlap in a projection image. These crossing points are not invariant landmarks on different mammograms. Because of the elasticity of the breast tissue, there is large variability in the positioning and compression used in mammographic examination. As a result, the relative positions of the breast tissues projected onto a mammogram vary from one examination to the other. Techniques that depend on identification of control points may not be generally applicable to registration of breast images.

Gopal et $a .^{8-10}$ and Hadjiiski et al. ${ }^{11}$ have developed a multistage technique that defines the transformation to locally map the position of the mass on a current mammogram to that of the prior mammogram. A local search for the mass is then performed on the prior mammogram. Good et al. ${ }^{12}$ also have developed a technique that defines a transforma- 


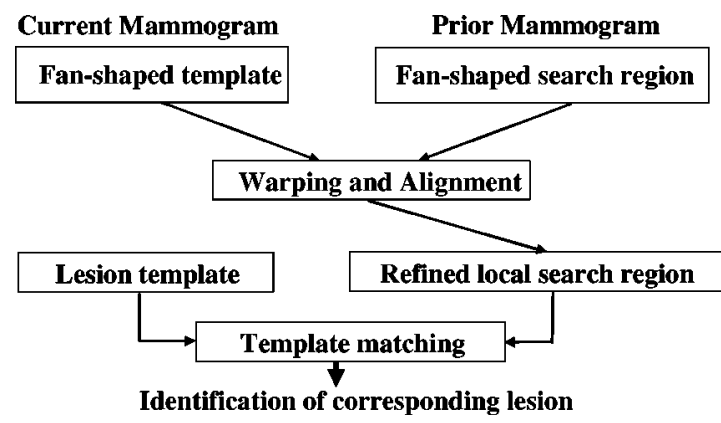

FIG. 1. Block diagram of the regional registration technique.

tion to map all points from the current mammogram onto a prior mammogram. The current mammogram is then subtracted from the prior mammogram.

The goal of our research is to develop a technique for computerized analysis of temporal differences between a mass on the most recent mammogram and a prior mammogram of the same view. The computer algorithm will assist radiologists in quantifying interval changes and thus distinguishing between benign and malignant masses for CAD. When fully developed, the technique will be applied to a mass on the current mammogram either identified by the radiologist or by an automated mass detection program, thus the interval change analysis can be an integrated part of an automated CAD system. In this study, we focused on the development of an automated registration technique that localizes the corresponding mass on the prior mammogram when the mass on the current mammogram is known. Therefore, we used radiologist-identified mass location on the current mammogram as a starting point and that on the prior mammogram as the ground truth for evaluation of the registration technique. A local registration technique was developed based on an affine transformation and simplex optimization and its usefulness in improving the localization of the mass on the prior mammogram was investigated.

\section{REGISTRATION TECHNIQUE}

A multistage regional registration technique was developed for identifying corresponding masses on temporal pairs of mammograms. The block diagram of the regional registration technique is shown in Fig. 1. In the first stage, an initial fan-shaped search region was defined on the prior mammogram based on the mass location on the current mammogram. In the second local alignment stage, the location of the search region on the prior mammograms was first refined by maximizing a correlation measure between a template of the fan-shaped region centered at the mass extracted from the current mammogram and the breast structures on the prior mammogram. The affine transformation in combination with simplex optimization was then employed to warp this local region and further improve the correlation. In the final stage, a search for the best match between the lesion template from the current mammogram and a structure on the prior mammogram was carried out within the refined

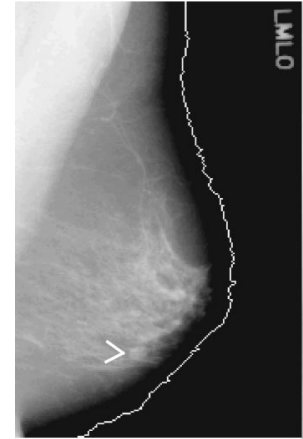

Current (1996)

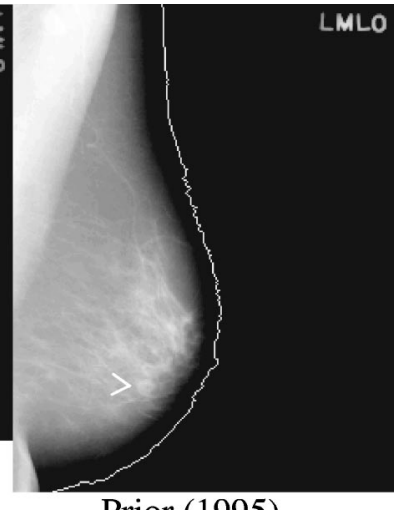

Prior (1995)
FIG. 2. An example of a pair of current and prior mediolateral oblique mammograms in our data set. The arrows point to the masses on the current and the prior mammograms. The white lines represent the breast boundary determined by the automated boundary detection procedure.

search region. A more detailed explanation for each of the stages will be presented in the following subsections.

\section{A. Stage 1-Initial estimate of search region}

We have modified our previous method to define a fanshaped search region on the prior mammogram. Initially an automated procedure is used to detect the breast boundary on the mammograms (Fig. 2). The location of the mass on the current mammogram is determined in a polar coordinate system with the nipple as the origin. By using the radial distance $R_{\text {curr }}$ between the nipple and mass centroid, $|\mathbf{N M}|$, an arc is drawn which intersects the breast boundary at points $\mathbf{A}$ and B (Fig. 3). Three angles are estimated at the radial distance $R_{\text {curr }}$ : The angle $\beta$ between $\mathbf{N M}$ and NA, the angle $\varphi$ between NM and $\mathbf{N B}$, and the angle $\theta$ between NA and NB $(\theta=\beta+\varphi)$. The location of the mass is determined by $R_{\text {curr }}$ and the angle $\beta$ or $\varphi$. The angle $\theta$ is the breast width at the radial distance $R_{\text {curr }}$. Using the radial distance $R_{\text {curr }}$ to draw an arc centered at the nipple centroid on the prior mammogram, $\mathbf{N}^{\prime}$, the two intersect points $\mathbf{A}^{\prime}$ and $\mathbf{B}^{\prime}$ with the breast boundary on the prior mammogram are determined. The

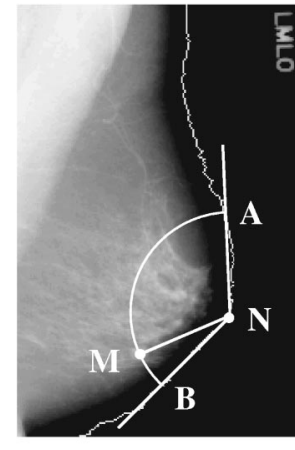

Current

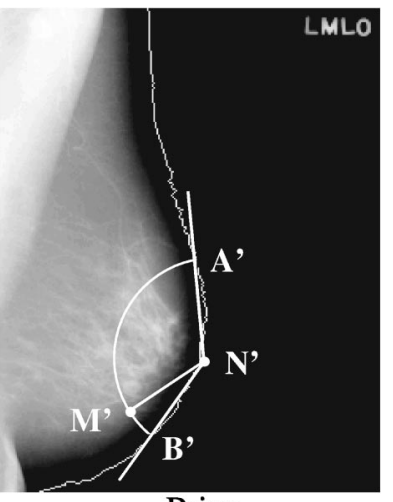

Prior
FIG. 3. Initial estimation of the mass location on the prior mammogram, based on the nipple-mass centroid distance and an angular distance from the breast periphery on the current mammogram. 


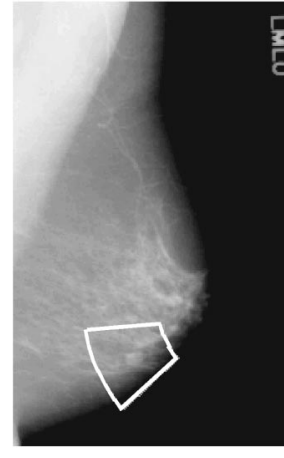

Current

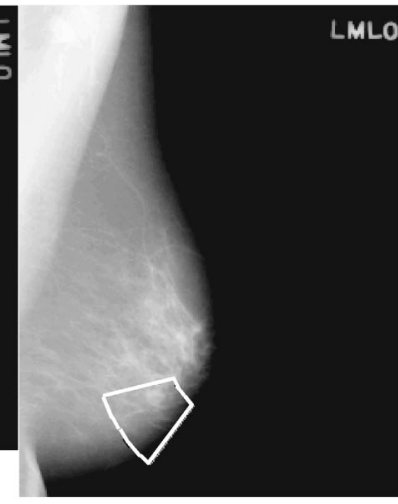

Prior
FIG. 4. Definition of an initial fan-shaped search region on the prior mammogram and a fan-shaped template on the current mammogram.

angle $\theta_{p}$ between the axes $\left|\mathbf{N}^{\prime} \mathbf{A}^{\prime}\right|$ and $\left|\mathbf{N}^{\prime} \mathbf{B}^{\prime}\right|$ is estimated. An angular scaling factor $\alpha$ can be calculated as the ratio of the prior and the current angles, $\alpha=\theta_{p} / \theta$.

In order to predict the angular location of the mass on the prior mammogram, the smaller angle between $\beta$ and $\varphi$ is selected as the angular coordinate of the mass on the current mammogram. The smaller angle is used because we found by experiment that it produces a smaller angular deviation error than using the larger angle. The angular deviation error is defined as the angle between the axis connecting the nipple and the true mass centroid and the axis connecting the nipple and the predicted mass centroid on the prior mammogram. The selected angle, multiplied by the angular scaling factor $\alpha$, is used as the predicted angle from the corresponding axis on the prior mammogram. The radial distance $R_{\text {curr }}$ is used to predict the radial position of the mass on the prior mammogram.

An initial fan-shaped search region is then defined on the prior mammogram centered at the predicted location of the mass centroid (Fig. 4). The size of the fan-shaped region is estimated previously ${ }^{10}$ to have the form $\epsilon=k_{1}+k_{2} / R_{\text {curr }}$ and $\delta=k_{3}$, where $2 \epsilon$ determines the angular width and $2 \delta$ determines the radial length of the fan-shaped region. The constants $k_{1}, k_{2}$, and $k_{3}$ were chosen experimentally such that the estimated fan-shaped regions will essentially include all mass centroids on the prior mammograms. A fan-shaped template centered at the mass is also defined on the current mammogram. More details on defining the fan-shaped region can be found in Appendix A and in Ref. 10.

\section{B. Stage 2-Refinement of search region by warping and alignment}

The second stage combined two procedures. First, the location of the search region on the prior mammograms was refined by maximizing a correlation measure between the fan-shaped template extracted from the current mammogram and the breast structures on the prior mammogram. The template was shifted pixel by pixel within the initial fan-shaped search region and a correlation measure was calculated at each pixel location. The pixel location providing the maxi-

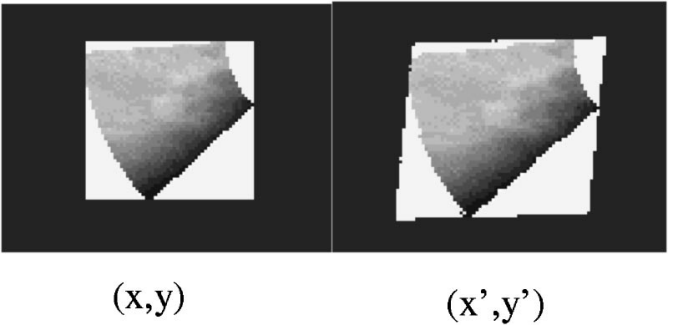

FIG. 5. The fan-shaped template $(x, y)$ and the warped fan-shaped template $\left(x^{\prime}, y^{\prime}\right)$ by the affine transformation.

mum correlation is used as the center of a refined search region. This is basically a template matching operation. Second, the affine transformation in combination with simplex optimization was iteratively used to warp the fan-shaped template and further maximize the correlation measure with the breast structures on the prior mammogram.

\section{Affine transformation}

An affine transformation ${ }^{13}$ is a linear transformation combining scaling, rotation, and translation. A two-dimensional affine transformation is defined as follows:

$$
\begin{aligned}
& x^{\prime}=a x+b y+c, \\
& y^{\prime}=d x+e y+f,
\end{aligned}
$$

where $(x, y)$ are the original coordinates, $\left(x^{\prime}, y^{\prime}\right)$ are the transformed coordinates, and $a, b, d, e, c, f$ are the transformation coefficients. The coefficients $a, b, d, e$ determine a scaling and a rotation, and the coefficients $c$ and $f$ determine a translation. The result of applying the affine transformation of Eq. (1) in combination with the simplex optimization (described below) to refine the fan-shaped search region is shown in Fig. 5. Since the affine transformation is linear, the transformed object is linearly resized and rotated. This can be observed from the edges of the bounding box of the fanshaped region (white box in Fig. 5). After the transformation the edges are still straight lines, however, the corner angles are different from 90 degrees and the lengths of the lines are linearly scaled.

\section{Nonlinear simplex optimization}

The nonlinear simplex optimization by Nelder and $\operatorname{Mead}^{14,15}$ is used to adjust the coefficients $a, b, c, d, e$, and $f$ and to warp the fan-shaped template, thereby maximizing the correlation between the template and a breast structure on the prior mammogram. This optimization defines a hyper-polygon. For each vertex an error function is calculated. The polygon is then "rolled" towards the minimum. The movement of the polygon (towards the minimum) is obtained by reflection in the direction opposite to the vertex with the maximal error. Figure 5 shows the result of application of the affine transformation whose coefficients were obtained by the nonlinear simplex optimization. A more detailed discussion on this optimization method can be found in Appendix B and Refs. 14 and 15. 


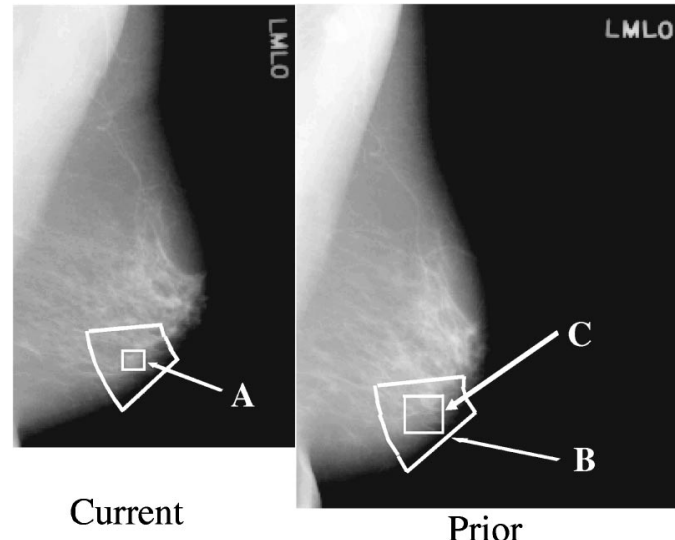

FIG. 6. A refined search region was defined on the prior mammogram. A search for the best match between the mass template from the current mammogram and a structure on the prior mammogram was carried out within the refined search region. (A-mass template on current mammogram, B-warped fan-shaped region from current mammogram, C-refined search region)

\section{Stage 3-Mass template matching and localization of corresponding lesion}

At this stage a new search region with a reduced size is defined on the prior mammogram (Fig. 6). The reduced size of the search region is determined experimentally by iterative adjustment of the size of the rectangular region targeting the improvement of the final result. A template containing the mass is extracted from the current mammogram. The mass location on the prior mammogram is then determined by maximizing the correlation between the template and a structure within the search region (Fig. 7).

\section{DATA SET}

A set of 124 temporal pairs of mammograms containing biopsy-proven masses on the current mammograms was used to examine the performance of this approach. Different mammographic views of the same breast were also included. There were a total of 221 mammograms obtained from 54 cases. Temporal pairs were formed using the temporal se-

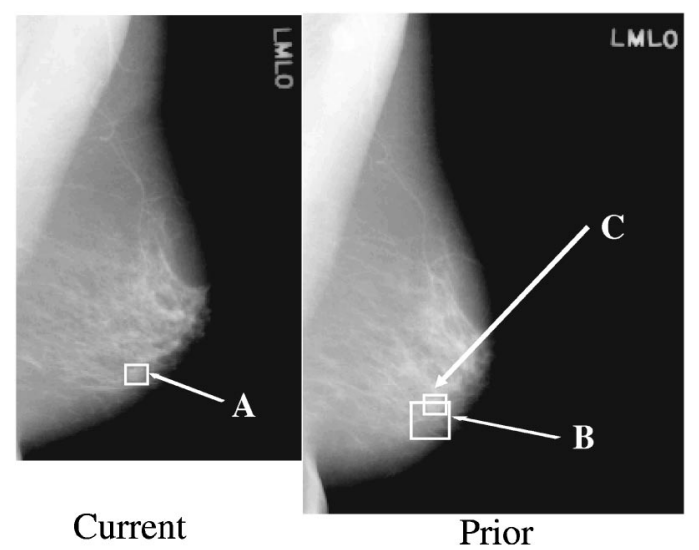

FIG. 7. Final identification of the corresponding mass on the prior mammogram. (A-Mass template on current mammogram, B-Refined search region, $\mathrm{C}$-Identified mass location). quence from the corresponding view. Some cases contained mammograms of multiple years and a combination of the mammograms from different prior years with the currentyear mammogram formed multiple temporal pairs. Thirty five of the mammograms were digitized with a LUMISYS DIS-1000 laser scanner at a pixel resolution of $100 \mu \mathrm{m} \times 100$ $\mu \mathrm{m}$ and 4096 gray levels. The digitizer was calibrated so that gray level values were linearly proportional to the optical density (OD) within the range of 0.1-2.8 OD units, with a slope of $0.001 \mathrm{OD} /$ pixel value. Outside this range, the slope of the calibration curve decreased gradually. The OD range of the digitizer was $0-3.5$. The remaining 186 mammograms were digitized with a LUMISCAN 85 laser scanner at a pixel size of $50 \mu \mathrm{m} \times 50 \mu \mathrm{m}$ and 4096 gray levels. The digitizer was calibrated so that the gray level values were linearly proportional to the OD within the range of 0-4 OD units, also with a slope of $0.001 \mathrm{OD} /$ pixel value. Output from both digitizers was linearly converted so that large pixel value corresponded to a low-optical density. In order to process the mammograms digitized with these two different digitizers, the images were first averaged using a filter that has constant weights over the entire filter kernel and then were downsampled. This filter will be referred to as a box filter. The images digitized with the LUMISCAN 85 digitizer were averaged with a $16 \times 16$ box filter and then were down-sampled by a factor of 16 . The images digitized with the LUMISYS DIS-1000 digitizer were averaged with an $8 \times 8$ box filter and then were down-sampled by a factor of 8 . Therefore, all resulting images had a pixel size of $800 \mu \mathrm{m} \times 800 \mu \mathrm{m}$.

The 54 cases contained 53 biopsy proven and one follow-up masses. The 221 mammograms contained different mammographic views and multiple years of the masses including the year when the biopsy was performed. Of the 124 temporal pairs of mammograms 73 were malignant and 51 benign. A malignant temporal pair consists of a biopsy proven malignant mass or a mass that was followed up and was found to be malignant when a biopsy was performed in a future year. Of the 124 temporal pairs of mammograms, 63 were CC-view pairs, 48 were MLO-view pairs, and 13 were lateral-view pairs. A Mammography Quality Standards Act (MQSA)-approved radiologist read the original mammogram to identify the mass and provide description of its characteristics. The radiologist defined a bounding box around the mass and marked the nipple location on every film.

The radiologist also measured the mass sizes, defined as the longest dimension of the mass, both on the current and prior mammograms. In Figs. 8(a) and 8(b) the mass sizes on the current mammograms were plotted against those on the prior mammograms for the malignant and the benign temporal pairs, respectively. Only 103 temporal pairs were plotted (54 malignant and 49 benign) due to the fact that the masses on the prior mammograms in the remaining 21 temporal pairs were too subtle for the radiologist to estimate their boundaries. On average the malignant masses appear to have a larger increase in size than the benign masses. The mean increase in size from prior to current for the malignant masses is $4.2 \mathrm{~mm}$ compared to $1.6 \mathrm{~mm}$ for the benign masses $(p=0.008)$. The correlation coefficient is 0.71 for the malig- 


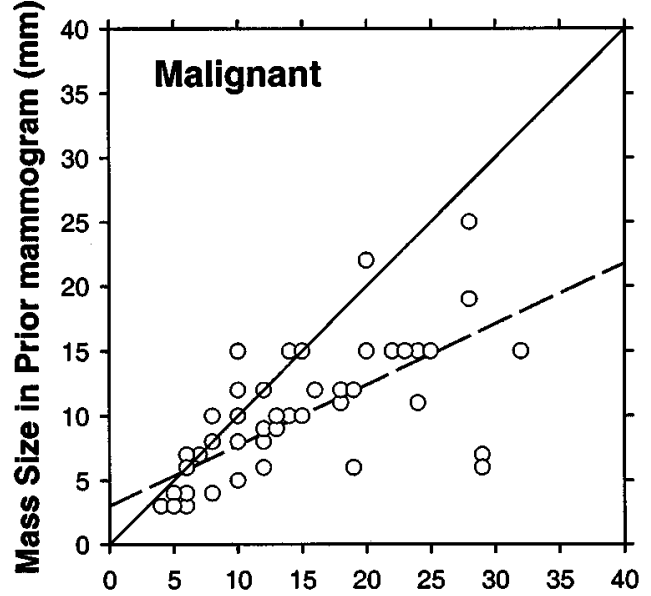

Mass Size in Current mammogram (mm)

(a)

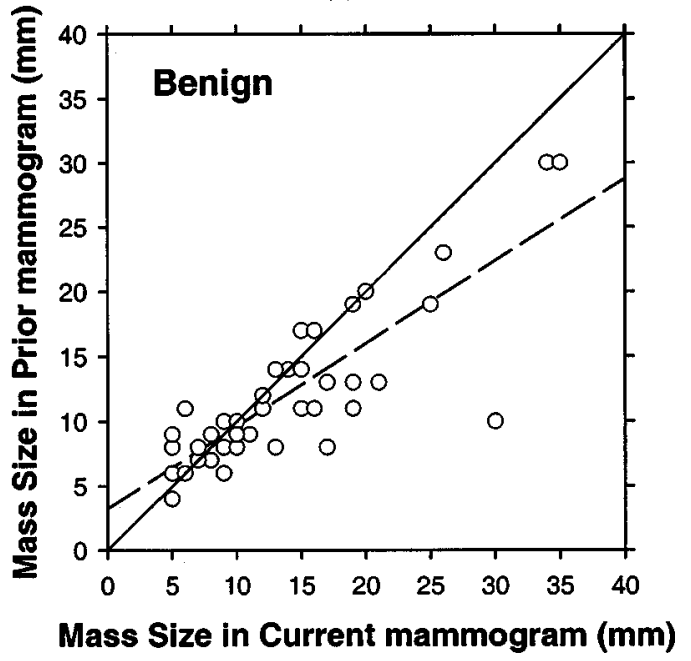

(b)

FIG. 8. Mass sizes measured by an MQSA-approved radiologist on the current mammograms plotted against those on the prior mammograms for (a) 54 malignant and (b) 49 benign temporal pairs. The diagonal line on the graph represents the case when the current and the prior mass sizes are identical. The dashed lines are the linear regression lines defined by $y$ $=0.469 x+3.012$ for (a) and by $y=0.638 x+3.242$ for (b). The correlation coefficient for malignant masses is 0.71 and for benign masses is 0.83 .

nant masses and 0.83 for the benign masses [Fig. 8(a) and $8(\mathrm{~b})]$.

The radiologist also rated the visibility of the masses on the mammograms relative to those encountered in clinical practice on a 10-point scale, with one represents the most obvious and 10 the subtlest masses. The visibility of the masses on the current mammogram is plotted against those on the prior mammogram in Fig. 9 for the 73 malignant and 51 benign temporal pairs. Generally, the malignant masses were less visible on the prior mammograms while the visibility of the benign masses was found to be more similar. The mean difference in visibility between the prior and the current mammograms for the malignant masses is 2.8 compared to $0.7 \mathrm{~mm}$ for the benign masses $(p=0.0002)$. The correlation coefficient is 0.06 for malignant masses and 0.54 for benign masses [Figs. 9(a) and 9(b)]. For most of the

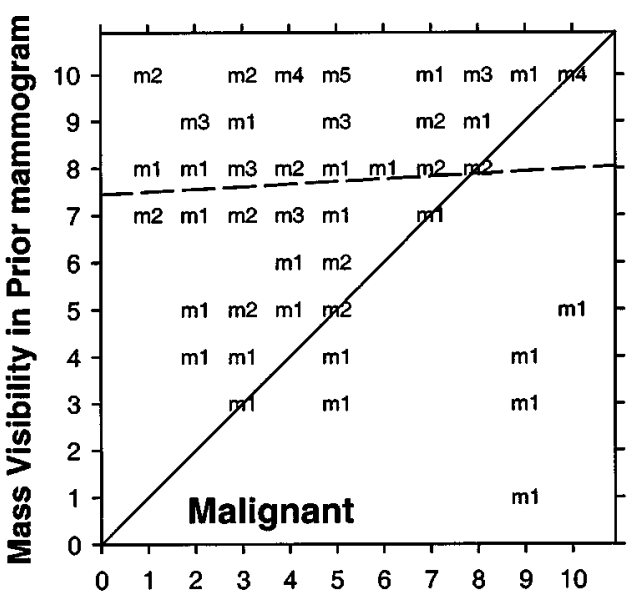

Mass Visibility in Current Mammogram

(a)

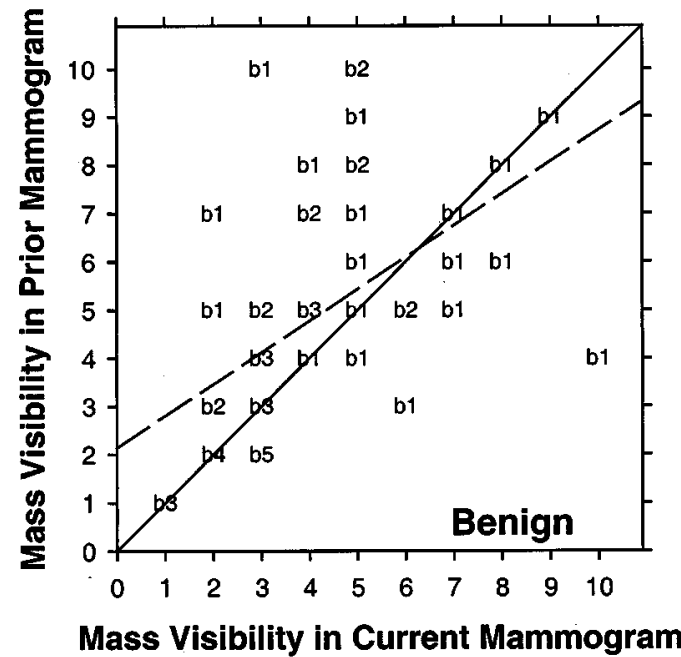

(b)

FIG. 9. Visibility of the masses on the current mammogram plotted against those on the prior mammogram for (a) malignant and (b) benign temporal pairs. The visibility was rated on a 10 -point discrete scale $(1=$ most obvious, $10=$ subtlest). Because many of the data points overlap, we indicate the number of points with the same rating by a number next to the symbol ( $m$ or $b$ ). The diagonal line on the graph represents the case when the current and the prior mass sizes are identical. The dashed lines are the linear regression lines defined by $y=0.055 x+7.44$ for (a) and by $y=0.658 x+2.138$ for (b). The correlation coefficient for malignant masses is 0.06 and for benign masses is 0.54 .

temporal pairs the time interval between the current and the prior mammogram was 12 months (Fig. 10).

\section{EVALUATION METHODS}

The accuracy of the multistage regional registration was analyzed in terms of two measures. The first measure is the overlap area between the estimated and the true lesions on the prior mammogram. The fractions of registered temporal pairs that could provide an accuracy of over $50 \%$ area overlap and over $75 \%$ area overlap were examined. The second measure is the average Euclidean distance between the centroids of the estimated and the true lesion locations. 


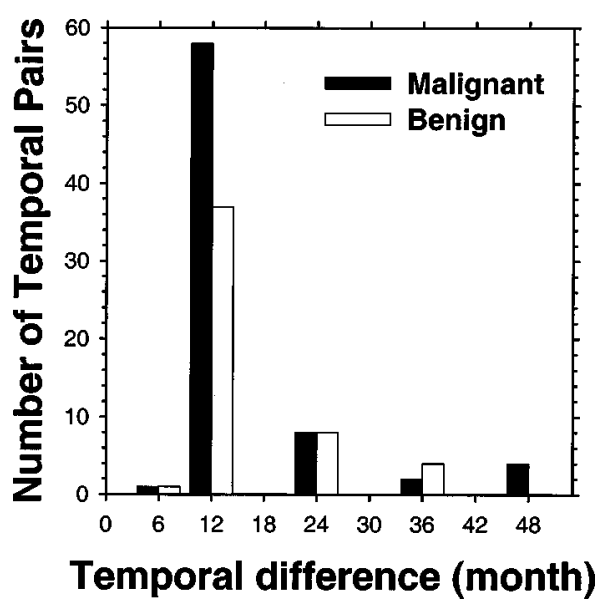

FIG. 10. Temporal interval between the current and the prior mammograms for the 124 temporal pairs in our data set.

\section{REGISTRATION RESULTS}

\section{A. Stage 1-Initial estimate of search region}

At this stage an initial estimation of the mass location on the prior mammogram was carried out based on the geometrical position of the mass on the current mammogram. Based on observation of the radial deviation errors and the angular deviation errors, the fan-shaped search region was estimated to be $\epsilon=0.25+5 / R_{\text {curr }}$ radians and $\delta=20 \mathrm{~mm}$. This definition of the fan-shaped search region resulted in an average search area of $1462 \mathrm{~mm}^{2}$ on the prior mammograms. For the 124 temporal image pairs used in this study, the Euclidean distance between the initial estimate of the centroid location of the corresponding structure on the prior mammogram and the center of the bounding box of the mass provided by the radiologist was estimated. For the 124 temporal image pairs, the average Euclidean distance error of the initial estimate was $8.4 \pm 5.4 \mathrm{~mm}$. The error distributions for both the malignant and the benign pairs are shown in Fig. 11. At this initial stage, $57 \%$ of the estimated lesion locations resulted in an area overlap of at least 50\% with the true lesion locations and 27\% resulted in an area overlap of at least $75 \%$ (Fig. 12).

\section{B. Stage 2-Refinement of search region by warping and alignment}

At the second stage, the location of the search region on the prior mammogram was first refined by maximizing a correlation measure between the fan-shaped template extracted from the current mammogram and the breast structures on the prior mammogram. The affine transformation in combination with simplex optimization was then employed to warp this local region. For the 124 temporal image pairs, the average Euclidean distance error after the second stage was $7.5 \pm 5.4 \mathrm{~mm}$. At this stage, $59 \%$ of the estimated lesion locations resulted in an area overlap of at least $50 \%$ with the true lesion locations, and 36\% resulted in an area overlap of at least $75 \%$. The average Euclidean distance error at this

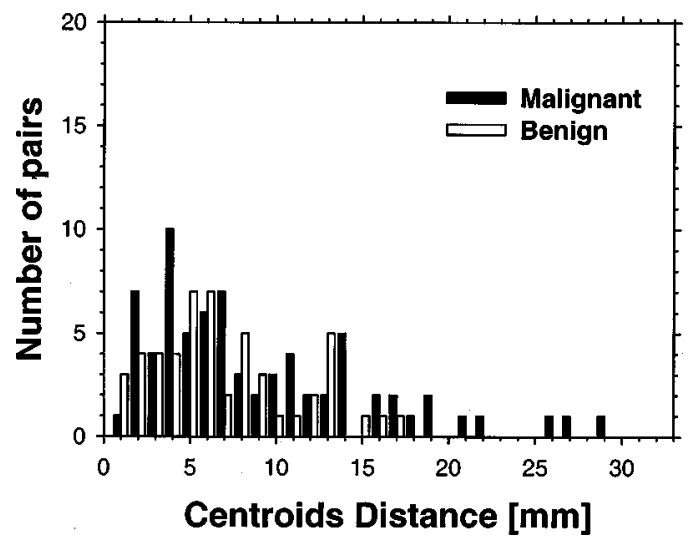

FIG. 11. Distribution of Euclidean distance error between the initial estimate of the mass centroid location on the prior mammogram and the center of the bounding box of the mass provided by the radiologist for the malignant and benign pairs after the first detection stage.

stage was reduced compared to that of the first stage, however, it did not achieve statistical significance $(p=0.07)$.

After the simplex optimization, the search region was reduced to a constant size of $24 \mathrm{~mm} \times 24 \mathrm{~mm}\left(=576 \mathrm{~mm}^{2}\right)$ centered at the refined fan-shaped region for every prior mammogram.

\section{Stage 3-Mass template matching and localization of corresponding lesion}

At this final stage, a search for the best match between the lesion template from the current mammogram and a structure on the prior mammogram was carried out within the refined search region. This template matching resulted in $87 \%$ of the estimated lesion locations having an area overlap of at least $50 \%$ with the true lesion locations. The distributions of the Euclidean error for the malignant and the benign temporal pairs are shown in Fig. 13. The average distance between the estimated and the true centroids of the lesions on the prior mammogram for all 124 pairs was $4.2 \pm 5.7 \mathrm{~mm}$ with a maximum of $31.6 \mathrm{~mm}$. These results are summarized in Table I. For the $87 \%$ of the temporal pairs with $50 \%$ overlap, the

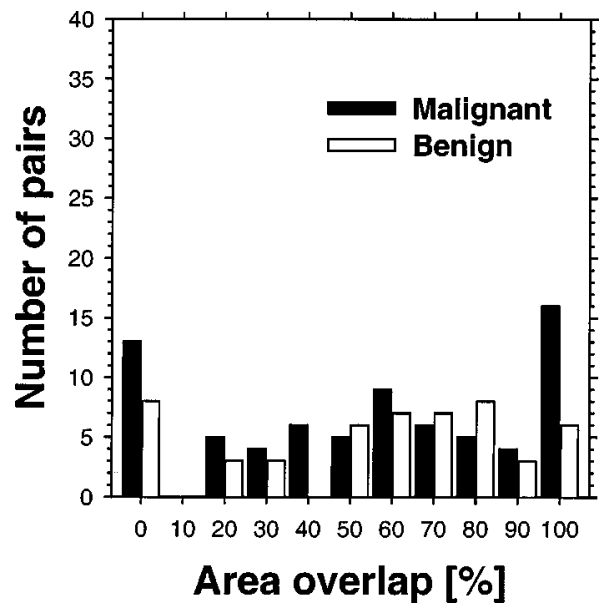

FIG. 12. Distribution of the area overlap between the estimated and the true lesion locations for 124 temporal pairs after the first detection stage. 


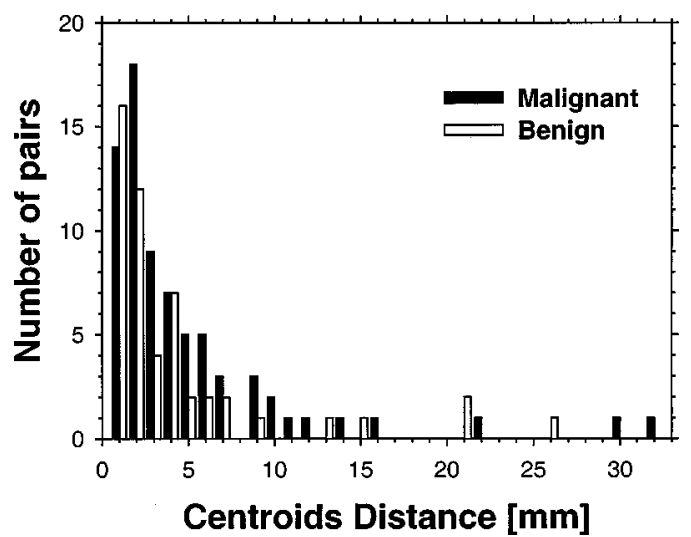

FIG. 13. Distribution of Euclidean distance error between the estimate of the mass centroid location on the prior mammogram and the center of the bounding box of the mass provided by the radiologist for the malignant and benign pairs after the final detection stage.

average distance between the estimated and the true centroids of the lesions on the prior mammogram was $2.4 \pm 2.1$ $\mathrm{mm}$ with a maximum of $10.2 \mathrm{~mm}$. When a more stringent criterion of $75 \%$ overlap is imposed, $82 \%$ of the masses on the prior mammograms are considered to be localized (Fig. 14). For the $82 \%$ of the temporal pairs with $75 \%$ overlap, the average distance between the estimated and the true centroids of the lesions on the prior mammogram was $2.2 \pm 1.9$ $\mathrm{mm}$ with a maximum of $10.2 \mathrm{~mm}$. The average Euclidean distance error at this stage was significantly reduced compared to the error of the first stage $(p=0.000001)$ and the error of the second stage $(p=0.000001)$.

\section{Study of the importance of the stage 2 procedures}

The effect of the two procedures at Stage 2 on the registration accuracy was studied. We removed them one at a time and evaluated the registration results. When the first correlation procedure was removed, the average Euclidean distance error increased to $5.6 \pm 8.2 \mathrm{~mm}$ in the final stage. Only $81 \%$ of the estimated lesion locations resulted in an area overlap of at least $50 \%$ with the true lesion locations and $75 \%$ resulted in an area overlap of at least $75 \%$ with the true lesion locations. When the second warping procedure was removed, the average Euclidean distance error increased to $5.0 \pm 6.3 \mathrm{~mm}$ in the final stage. Only $82 \%$ of the estimated

TABLE I. The Euclidean distance between the true and the estimated centroids of the mass on the prior mammogram for the three detection stages.

\begin{tabular}{llrrr}
\hline \hline & & Overall & $50 \%$ overlap & $75 \%$ overlap \\
\hline \multirow{4}{*}{ Stage 1 } & Mean distance & $8.4 \mathrm{~mm}$ & $5.6 \mathrm{~mm}$ & $4.5 \mathrm{~mm}$ \\
& Standard. Deviation. & $5.4 \mathrm{~mm}$ & $2.8 \mathrm{~mm}$ & $2.6 \mathrm{~mm}$ \\
& Max. distance & $29.0 \mathrm{~mm}$ & $16.2 \mathrm{~mm}$ & $13.8 \mathrm{~mm}$ \\
& Mean distance & $7.5 \mathrm{~mm}$ & $4.9 \mathrm{~mm}$ & $3.9 \mathrm{~mm}$ \\
Stage 2 & Standard. Deviation. & $5.4 \mathrm{~mm}$ & $3.0 \mathrm{~mm}$ & $2.6 \mathrm{~mm}$ \\
& Max. distance & $32.0 \mathrm{~mm}$ & $16.9 \mathrm{~mm}$ & $11.6 \mathrm{~mm}$ \\
& Mean distance & $4.2 \mathrm{~mm}$ & $2.4 \mathrm{~mm}$ & $2.2 \mathrm{~mm}$ \\
Stage 3 & Standard. Deviation & $5.7 \mathrm{~mm}$ & $2.1 \mathrm{~mm}$ & $1.9 \mathrm{~mm}$ \\
& Max. distance & $31.6 \mathrm{~mm}$ & $10.2 \mathrm{~mm}$ & $10.2 \mathrm{~mm}$ \\
\hline \hline
\end{tabular}

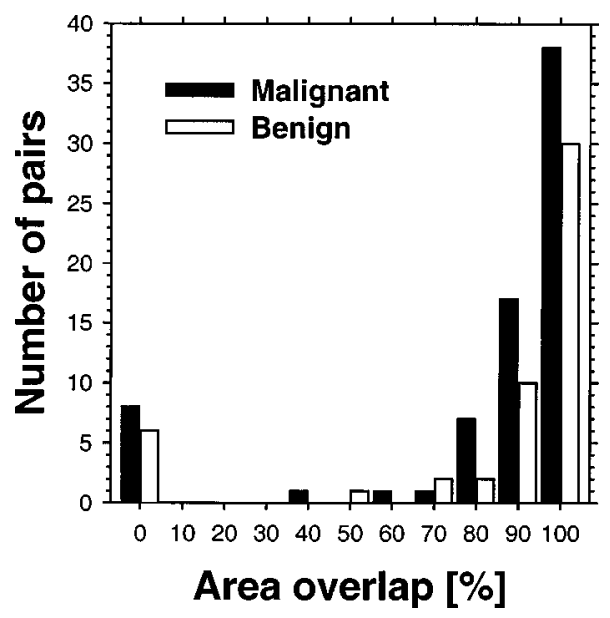

FIG. 14. Distribution of the area overlap between the estimated and the true lesion locations for 124 temporal pairs after the final detection stage.

lesion locations resulted in an area overlap of at least 50\% with the true lesion locations and $76 \%$ resulted in an area overlap of at least $75 \%$ with the true lesion locations.

\section{DISCUSSION}

The approach proposed here has simplified the first stage compared to our previous method. ${ }^{10}$ In the previous method, the distances between the nipple and the breast centroid on the current and prior mammograms were determined and used to estimate a radial scaling factor. The angular location of the mass was measured from the nipple-breast centroid axis. A global alignment procedure was used for determination of the breast centroids. With our new approach we eliminated the scaling for the radial distance between the nipple and the mass location of the prior mammogram. The breast periphery was used as a reference for the estimation of the angular position of the mass. Therefore, there was no need to determine the breast centroids on the current and the prior mammograms and the global alignment procedure could be eliminated. This is possible because the local alignment step provides better compensation for the displacement of the corresponding masses on the current and the prior mammogram caused by different compression and positioning of the breast.

It was found that the estimation of the angular position from the breast periphery allowed more precise localization of the mass position on the prior mammogram compared to our previous method where the angular position of the mass was estimated based on the nipple-breast centroid axis. ${ }^{10}$ There is a large variability in the estimation of the breast centroid location because the extend of the breast imaged on the mammogram at the chest wall and at the axillary tail in the MLO view depends on the breast positioning and compression. This causes an uncertainty in defining the region to calculate the breast centroid. In the previous study using 74 temporal pairs, the estimated Euclidean distance error at the first stage was $9.8 \pm 6.0 \mathrm{~mm}$. The fan-shaped search region was defined as $\epsilon=0.35+5 / r$, resulting in an average area of $1865 \mathrm{~mm}^{2}$ for the fan-shaped search region. In the current 

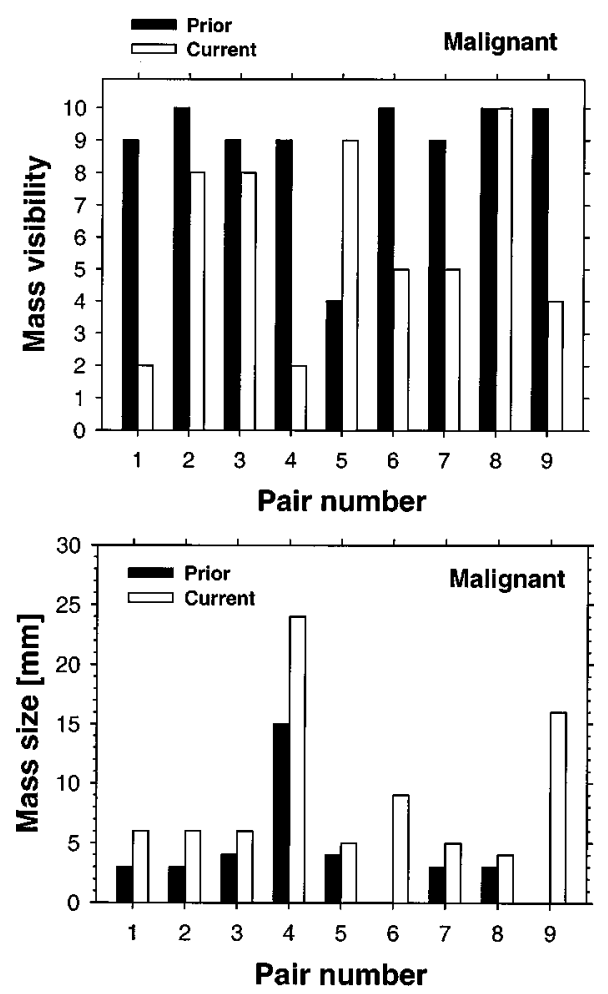

FIG. 15. The visibility and the mass size of nine malignant temporal pairs having area overlap less than $50 \%$. The radiologist was unable to define the prior mass sizes of pairs 6 and 9 due to the subtlety of these masses.

study, the estimated Euclidean distance error at the first stage was reduced to $8.4 \pm 5.4 \mathrm{~mm}$ even though the data set was increased to 124 temporal pairs of mammograms. This allows the fan-shaped region to be reduced to $\epsilon=0.25+5 / r$, resulting in an average fan-shaped search area of $1462 \mathrm{~mm}^{2}$ on the prior images. The reduction of the search area improves the chance of correctly localizing the mass on the prior mammogram.

The second stage combined two procedures: First the localization of the search region on the prior mammograms was refined by maximizing a correlation measure between the fan-shaped template extracted from the current mammogram and the breast structures on the prior mammogram. The affine transformation in combination with simplex optimization was then employed to warp and locally align the template with the breast structures. Both procedures improved the detection process. When one of these procedures was removed the registration results deteriorated, as discussed in the Results section.

With these improvements, the accuracy of the current regional registration technique is improved over the previous method. ${ }^{10}$ The current technique produced an average Euclidean distance error of $4.2 \pm 5.7 \mathrm{~mm}$, compared to $5.4 \pm 7.5$ $\mathrm{mm}$ when the previous technique was applied to the current data set. This difference is statistically significant $(p=0.03)$. $82 \%$ of the estimated lesion locations resulted in an area overlap of at least $75 \%$ with the true lesion locations compared with $72 \%$ when applying the previous technique to the current data set. It is interesting to note that, of the 21
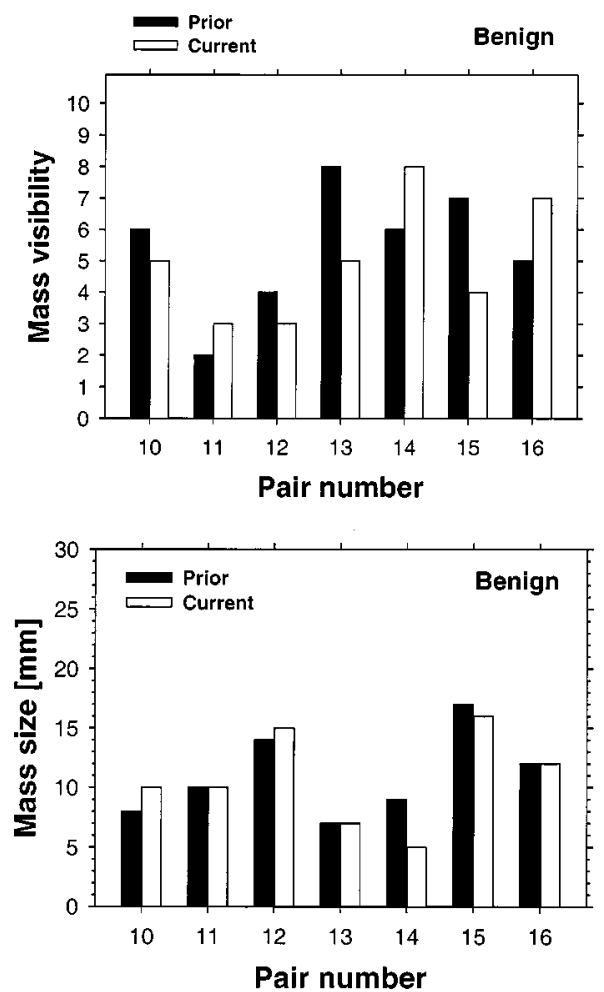

FIG. 16. The visibility and the mass size of seven benign temporal pairs having area overlap less than $50 \%$.

"masses" on the prior mammograms that the experienced radiologist could not confidently define the mass and measure its size, our registration technique localize 19 of them with an area overlap greater than $50 \%$.

The average distance between the estimated and the true centroid of the lesions on the prior mammogram for the subset of temporal pairs having 50\% overlap is about half of that of the entire data set (Table I). The maximum distance for this subset is about $1 / 3$ of that for the entire data set.

With the current regional registration technique, 16 temporal pairs (13\% of 124 temporal pairs) have an area overlap less than 50\%. Twelve of the 16 computer estimated locations do not overlap at all with the radiologist's identified locations, and the other four pairs have an overlap between $1 \%$ and $49 \%$. Seven of them are benign and nine are malignant. A major cause of the misregistration was that the mass was small and subtle and a breast structure within the search region had a higher correlation with the mass template from the current mammogram. Figures 15 and 16 show the visibility ratings and sizes of these misregistered masses. Eight of the nine misregistered malignant masses have visibility ratings of 9 or 10 and sizes below $5 \mathrm{~mm}$. The misregistered benign masses are somewhat more obvious and larger in sizes than the malignant ones. Since many of the masses on the prior mammograms were not interpreted as a mass without reference to the current mammograms, the automatic registration with template matching would be difficult with these masses if the search region contains normal, but dense breast structures. We are currently investigating the application of local mass detection in the search region to focus 
template matching to a few suspicious areas. Morphological and texture features will be extracted from the potential mass areas to provide additional matching information in the feature space.

The interval change analysis, when fully developed, will be one of the functions provided in an integrated CAD system. The mass on the current mammogram can be detected by an automated mass detection algorithm or identified by a radiologist. The CAD system will then analyze whether the mass is an existing or a newly developed lesion and will estimate its likelihood of malignancy. We are developing methods for characterization of malignant and benign masses based on analysis of interval changes in the mass features. ${ }^{16}$ Investigation of criteria to determine whether a mass exists on the prior mammogram is underway. If the mass is a newly developed lesion on the current mammogram, it will then undergo a single-exam analysis by the CAD system.

\section{CONCLUSION}

We are developing an automated registration technique for analysis of interval change of a mass from a previous mammographic exam to the current one. In this study we found that a local affine transformation in combination with nonlinear simplex optimization can improve the localization and reduce the size of the search region. With the improved method, $87 \%$ of the estimated lesion locations in 124 randomly selected temporal pairs resulted in an area overlap of at least $50 \%$ with the true lesion locations. When the threshold for correct localization was set to $75 \%$ area overlap, $82 \%$ of the temporal pairs still exceeded this threshold. The average distance between the estimated and the true centroids of the lesions on the prior mammogram over all pairs was 4.2 $\pm 5.7 \mathrm{~mm}$. The registration accuracy of the current method has been improved in comparison with that of our previous method $^{10}$ even though the data set was increased from 74 pairs to 124 pairs. This improvement is obtained mainly from the second stage affine transformation and simplex optimization. Additional studies are currently underway to develop a feature matching method to further improve lesion localization.

\section{ACKNOWLEDGMENTS}

This work is supported by a Career Development Award from the U.S. Army Medical Research and Material Command (DAMD 17-98-1-8211) (L.H.), a USPHS Grant CA 48129, a USAMRMC grant (DAMD 17-96-1-6254), and a USAMRMC Career Development Award DAMD 17-96-16012 (B.S.). The content of this publication does not necessarily reflect the position of the government and no official endorsement of any equipment or product of any companies mentioned in the publication should be inferred.

\section{APPENDIX A: DEFINITION OF THE FAN-SHAPED REGION ON THE PRIOR MAMMOGRAM}

Refer to Figs. 3 and 4, the fan-shaped region on the prior mammogram is drawn based on the nipple centroid on the prior mammogram, $\mathbf{N}^{\prime}$, as the center of the coordinate sys- tem. The two bounding arcs are drawn using the radial distances $R_{\text {curr }}+\delta$ and $R_{\text {curr }}-\delta$, both centered at $\mathbf{N}^{\prime}$. The two sides of the fan-shaped region are bounded by two radial lines that form angles $\epsilon$ and $-\epsilon$ with the line $\left|\mathbf{N}^{\prime} \mathbf{M}^{\prime}\right|$. Thus the initial fan-shaped search region is centered as the predicted location of the mass centroid $\mathbf{M}^{\prime}$ on the prior mammogram (Fig.4).

The constants $k_{1}, k_{2}$, and $k_{3}$ were chosen experimentally based on analysis of the angular deviation errors and the corresponding radial deviation errors for the 124 temporal pairs. The radial deviation error is defined as the difference between the predicted and the true distance of the mass from the nipple on the prior mammogram. The constants $k_{1}, k_{2}$ are obtained in such a way that $\epsilon$ is the smallest upper bound that can enclose all angular deviation errors for all radial distances $\left(R_{\text {cur }}\right)$ and all temporal pairs. The selection of the parametric form of $\epsilon$ was discussed in detail in Ref. 10. It reduced $\epsilon$ at larger $R_{\text {cur }}$. The constant $k_{3}$ was chosen to be equal to the maximum radial deviation error.

\section{APPENDIX B: SIMPLEX OPTIMIZATION}

An optimization problem can be defined as an error function that has to be minimized by iterative selection of the values of the function parameters $n$. We can define $n+1$ dimensional space, where $n$ dimensions (degree of freedom) correspond to the error function parameters, and one dimension is the error function itself. When the optimization function is calculated for all possible values of the $n$ parameters, and error surface in $(n+1)$-dimensional space will be obtained. Usually the error functions for the real world applications are complex and nonlinear and the corresponding error surfaces contain local minima.

The nonlinear simplex optimization by Nelder and Mead $^{14,15}$ defines a hyper-polygon with $n+1$ vertexes in a $(n+1)$ dimensional space. For each vertex the error function is calculated. The polygon is then "rolled" towards the minimum. The movement of the polygon (towards the minimum) is obtained by reflection in the direction opposite to the vertex $(\mathbf{K})$ with the maximal error. To achieve this the center of masses $(\mathbf{L})$ of the hyper-polygon vertexes is calculated. A line KL connects the center of the masses with the vertex with the maximal error. The new vertex $\left(\mathbf{K}^{\prime}\right)$ is obtained by central projection of the vertex $\mathbf{K}$ on the line $\mathbf{K L}$ with center $\mathbf{L}$ and $\left|\mathbf{K}^{\prime} \mathbf{L}\right|=t|\mathbf{K L}|$. The coefficient $t$ determines how far the new vertex will be projected and what the corresponding size of the hyper-polygon will be. The larger the hyper-polygon is, the easier it will avoid ("roll over") the local minima on the error surface. However, it will be difficult to get close to the global minimum if its size is too large. On the other hand, although a small hyper-polygon will allow it to get to a close proximity to the global minimum, it is more likely to be trapped in a local minimum. The magnitude of the coefficient $t$ is controlled adaptively by the Nelder and Mead algorithm. In case a large reduction in the error is detected for the new vertex, the magnitude of $t$ is increased. In case the error is found to be increased for the new vertex, the magnitude of $t$ is decreased. 
The this paper, the nonlinear simplex optimization by Nelder and Mead was used to adjust the coefficients $a, b, c$, $d$, $e$, and $f$ and to warp the fan-shaped template, thereby maximizing the correlation $(C)$ between the template and a breast structure on the prior mammogram. Therefore, the dimensionality of the space was 7: Six parameters to be adjusted and the error function to be minimized was defined as $1-C$.

a) Author to whom correspondence should be addressed. Telephone: (734) 647-8552. Fax: (734) 647-8557. Electronic mail: 1hadjisk@umich.edu

${ }^{1}$ H. C. Zuckerman, "The role of mammography in the diagnosis of breast cancer," in Breast Cancer, Diagnosis and Treatment, edited by I. M. Ariel and J. B. Cleary (McGraw-Hill, New York, 1987), pp. 152-172.

${ }^{2}$ L. Tabar and P. B. Dean, "The control of breast cancer through mammographic screening: What is the evidence," Radiol. Clin. N. Amer. 25, 993-1005 (1987)

${ }^{3}$ L. W. Bassett, B. Shayestehfar, and I. Hirbawi, "Obtaining previous mammograms for comparison: usefulness and costs," Amer. J. Roentgenology 163, 1083-1086 (1994).

${ }^{4}$ E. A. Sickles, "Periodic mammographic follow-up of probably benign lesions: results in 3183 consecutive cases," Radiology 179, 463-468 (1991).

${ }^{5}$ M. Sallam and K. Bowyer, "Detecting abnormal densities in mammograms by comparison with previous screenings," in Digital Mammography '96, edited by K. Doi, M. L. Giger, R. M. Nishikawa, and R. A. Schmidt (Elsevier, Amsterdam, 1996).

${ }^{6}$ D. Brzakovic, N. Vujovic, M. Neskovic, P. Brzakovic, and K. Fogerty, "Mammogram analysis by comparison with previous screenings," in Digital Mammography '96, edited by K. Doi, M. L. Giger, R. M. Nishikawa, and R. A. Schmidt (Elsevier, Amsterdam, 1996).
${ }^{7} \mathrm{~N}$. Vujovic and D. Brzakovic, "Establishing the correspondence between control points in pairs of mammographic images," IEEE Trans. Image Process. 6, 1388-1399 (1997).

${ }^{8}$ S. Sanjay-Gopal, H. P. Chan, B. Sahiner, N. Petrick, T. Wilson, and M. Helvie, "Evaluation of interval change in mammographic features for computerized classification of malignant and benign masses," Radiology 205(P), 216 (1997)

${ }^{9}$ S. Sanjay-Gopal, H. P. Chan, N. Petrick, T. Wilson, B. Sahiner, M. Helvie, and M. Goodsitt, "A regional registration technique for automated analysis of interval changes of breast lesions," Proc. SPIE 3338, 118-131 (1998).

${ }^{10}$ S. Sanjay-Gopal, H. P. Chan, T. E. Wilson, M. A. Helvie, N. Petrick, and B. Sahiner, "A regional registration technique for automated interval change analysis of breast lesions on mammograms," Med. Phys. 26, 2669-2679 (1999).

${ }^{11}$ L. Hadjiiski, H. P. Chan, B. Sahiner, N. Petrick, M. A. Helvie, and S. S. Gopal, "Automated identification of breast lesions in temporal pairs of mammograms for interval change analysis," Radiology 213(P), 229-230 (1999).

${ }^{12}$ W. Good, B. Zheng, Y. H. Chang, X. Wang, and G. Maitz, "Generalized procrustean image deformation for subtraction of mammograms," Proc. SPIE 3661, 1562-1573 (1999).

${ }^{13}$ L. Quan and T. Kanade, "Affine structure from line correspondence with uncalibrated affine cameras," IEEE Trans. Pat. Anal. Machine Intel. 19(8), 834-845 (1997).

${ }^{14}$ S. S. Rao, Optimization: Theory and Applications (Wiley, New York, 1979).

${ }^{15}$ Numerical Methods for Non-Linear Optimization, edited by F. A. Lootsma (Academic, New York, 1972).

${ }^{16}$ L. Hadjiiski, B. Sahiner, H. P. Chan, N. Petrick, M. A. Helvie, and M. Gurcan, "Computer-aided classification of malignant and benign breast masses by analysis of interval change of features in temporal pairs of mammograms," Radiology 217(P), 435 (2000). 